\title{
La Salud Pública desde la Perspectiva de Género
}

\author{
Franklin Solís Zúniga ${ }^{1}$
}

\section{RESUMEN}

La evaluación de la equidad de género en el acceso a servicios de salud es un aspecto que aún no se ha estudiado lo suficiente y que merece un análisis reflexivo desde distintas disciplinas relacionadas con la Salud Pública. Este artículo tiene como objetivo principal, mostrar una revisión actual del análisis del enfoque de Salud Pública basado en el Género. No se pretende ser exhaustivo proporcionando profundidad de análisis, sino más bien, reflejar la necesidad de la integración de la perspectiva de género para lograr la equidad en el acceso a los servicios de salud. Tomando en cuenta que una de las Funciones Esenciales de la Salud Pública (FESP), hace referencia a la promoción de la equidad en el acceso a la atención de salud, que incluye la evaluación y la promoción del acceso efectivo de todas/os las/os ciudadanas/os a los servicios de salud necesarios. El artículo recoge los aportes de algunos teóricos sobre lo que implica la integración de la perspectiva de género para lograr la equidad en salud. Asimismo, señala la escasez de información precisa sobre la magnitud del problema en sus diferentes manifestaciones, y sobre su impacto en la salud. Se concluye con un llamado a los profesionales de la Salud Pública y sectores interesados, a profundizar esta reflexión de cara a determinar con mayor precisión cuál debe ser su papel en el abordaje del problema.

Palabras clave: Género, Salud Pública, Desigualdad, Equidad.

Recibido: 01 de diciembre de 2015

Aceptado: 29 de enero de 2016

1 Psicólogo-Docente. Universidad Nacional Autónoma de Nicaragua. UNAN- Managua. Facultad Regional Multidisciplinaria de Estelí. FAREM- Estelí. Correo Electrónico: franksolis23@yahoo.com 


\section{Public Health from a Gender Perspective}

\section{SUMARY}

The evaluation of gender equality in the access to the health services is an aspect that has not been studied enough and worthy of considerate analysis from different disciplines related to the public health. The main objective of this article is to show a current review of the analysis of the public health approach based on gender. It is not intended to be exhaustive providing depth analysis, but rather to reflect the need of integration of the gender perspective to achieve the gender equality in the access to the health services. Considering that one of the Essential Public Health Functions (FESP), refers to the promotion of the gender equality in the access to the health care, which includes the evaluation and promotion of the effective access to all the citizens to the necessary health services. The article presents some theoretical contributions about what involves the integration of the gender perspective to achieve equality in the health care. It also notes the lack of precise information on the magnitude of the problem in its different manifestations, and its impact on the health care. It concludes with a request to the professionals of the public health and stakeholders, to expand the reflection in order to determine more precisely which should be its role in addressing the problem.

Keywords: Gender, Public Health, inequality, Equality 


\section{Reflexiones Teóricas y Magnitud del Problema}

Según Aldereguía, Núñez y Fernández (1995), la salud se define a través de un proceso dinámico que se extiende desde la mortalidad prematura como peor expresión de la enfermedad hasta el elevado nivel de bienestar físico, mental y social y la capacidad óptima de funcionamiento, como máxima expresión posible de salud. Este proceso está influenciado por factores biológicos, psicológicos y del ambiente físico y social que pueden producir daños a la salud, y que se expresan fundamentalmente en la percepción de enfermedad y el acceso a los servicios de salud.

La sexualidad contempla los elementos: género, erotismo, afecto, reproducción. Es decir el género es un elemento constituido en la categoría sexualidad. Weeks, J. (1998). Por tanto, tanto, el género como el sexo se relacionan con la salud y lo hacen de forma simultánea, ya que las personas no viven siendo de un género o de un sexo, sino de ambos a la vez (Krieger, 2003).

En vista de esto, la salud no puede ser tratada de la misma manera en hombres y mujeres, no sólo por las características biológicas, sino porque ambos desempeñan distintos roles, se les han asignado diferentes espacios para llevarlos a cabo, tienen distintos estilos de vida y responsabilidades y las circunstancias que moldean la existencia social de la mujer distan de ser las mismas que para el hombre. Es decir, viven de manera diferente, lo que conlleva a que las enfermedades y la exposición a riesgos tales como hábitos de alimentación, ambiente de trabajo, estrés cotidiano, entre otros, los coloquen en situaciones de salud diferentes (Castañeda 2007).

Las diferencias de sexo y de género determinan diferencias en los determinantes de la salud, la vulnerabilidad, la naturaleza, severidad y frecuencia de los problemas de salud, la forma en la que se perciben los síntomas, la utilización y la accesibilidad de los servicios sanitarios, el esfuerzo diagnóstico y terapéutico, el cumplimiento del tratamiento y de los mensajes preventivos y el pronóstico de los problemas de salud entre hombres y mujeres (Borrell y Artazcoz, 2008).

En consecuencia, desde el modelo social de salud (Salud Pública), el sistema sexo-género se presentará como el marco oportuno para comprender las desigualdades en salud entre mujeres y hombres (Cantero y Gálvez, 2007).

En este sentido, según Cantero (2007), si los servicios de salud quieren caracterizarse por su equidad y ser más eficientes, se precisa mayor sensibilidad respecto a los conceptos sexo y género. Pero, la base de evidencia internacional para las políticas y las prácticas profesionales sobre esta línea de investigación es limitada, y las confusiones conceptuales son bastante comunes.

Según la OMS (2007), el género se refiere a los conceptos sociales de las funciones, comportamientos, actividades y atributos que cada sociedad considera apropiados para los hombres y las mujeres. Las diferentes funciones $\mathrm{y}$ comportamientos pueden generar desigualdades de género, es decir, diferencias entre los hombres y las mujeres que favorecen sistemáticamente a uno de los dos grupos. A su vez, esas desigualdades pueden crear inequidades entre los hombres y las mujeres con respecto tanto a su estado de salud como a su acceso a la atención sanitaria.

Las inequidades en salud se refieren, en gran medida, a las oportunidades desiguales de ser saludable, por pertenecer a grupos sociales distintos, como mujeres, negros, campesinos. Estas inequidades se relacionan con cuatro afrontamientos diferentes: el estado de salud de la población, expresado en los perfiles epidemiológicos, en función de la presencia y frecuencia de los problemas de salud de cada sexo; la posibilidad de la utilización de los recursos y los 
servicios para satisfacer las necesidades de hombres y mujeres; la gestión de salud referida a la oportunidad de desarrollar responsabilidades y ocupar cargos de decisión en el sector, de acuerdo a sus expectativas y aspiraciones de los individuos; y por último, el financiamiento de la atención de salud (Braveran y Gruskin, 2003).

Según Whitehead (1992), La inequidad en salud hace referencia a desigualdades que son innecesarias y evitables pero que, además, se consideran injustas. Para esta autora, la equidad en salud significa, idealmente, que todos deben tener la oportunidad justa de alcanzar todo su potencial de salud y, más pragmáticamente, que nadie debe estar en desventaja a la hora de alcanzarlo, si ello se puede evitar.

En términos operacionales esta autora supone una reducción, al mínimo posible, de los diferenciales de salud y de acceso a los servicios sanitarios. A partir de ese punto, explícitamente pragmático, define el concepto de equidad por medio de dos antónimos: desigualdad e inequidad. Para el primer término (desigualdad), indica diferencias sistemáticas, inevitables y pertinentes entre los miembros de una población, mientras que para el segundo (inequidad) señala la existencia de variaciones no solo innecesarias y evitables, sino también injustas.

Asimismo, apunta que la equidad no significa que todos deban tener el mismo nivel de salud y consumir la misma cantidad de servicios y recursos, sino que deben considerarse las necesidades de cada uno. Para describir una situación como no equitativa o injusta, ésta debe ser examinada y juzgada en el contexto de lo que sucede en el resto de la sociedad. Desde esta visión, toda inequidad es una desigualdad pero no toda desigualdad es una inequidad, pues una inequidad es una desigualdad injusta y potencialmente evitable.

En la mayoría de las sociedades la mujer ha tenido un menor estatus social que el hombre, lo que se traduce en relaciones de poder desiguales. Por ejemplo, la mujer se encuentra en condiciones de inferioridad en la familia, la comunidad y la sociedad en general; tiene un menor grado de acceso a los recursos y de control sobre los mismos, y un menor peso que los hombres en la toma de decisiones. Todos estos factores han llevado a restar importancia a la salud de la mujer y a no prestarle la debida atención.

La atención a la salud de la mujer se ha venido centrando hasta ahora en los problemas que ésta sufre durante el embarazo y el parto. Sin embargo, el enfoque de Salud Pública basado en el género ha servido para comprender mejor los problemas sanitarios de la mujer y determinar formas de abordarlos en mujeres de todas las edades. Por ejemplo, sabemos hoy que las enfermedades cardiovasculares son una importante causa de mortalidad femenina. Sin embargo, no se reconoce suficientemente este hecho, lo que retrasa la búsqueda de tratamiento y el diagnóstico entre las mujeres. La identificación de diferencias de género en las enfermedades cardiovasculares, ha permitido formular estrategias de promoción de la salud y prevención más eficaces, lo que a su vez ha redundado en mejoras de la salud de la mujer en muchos países (OMS, 2007).

\section{Entonces, ¿por qué es necesaria la integración de la perspectiva de Género para lograr la equidad en Salud?}

De acuerdo con la OPS (S.F), esta perspectiva nos ayuda reconocer el impacto del género en:

- El estado y determinantes de salud para hombres y mujeres

- Los obstáculos de acceso a los servicios y recursos de salud

- El impacto de las políticas y programas de saludLa distribución del poder y remuneración del trabajo en el sector salud. 
Según la OMS (2007), el enfoque de Salud Pública basado en el género parte del reconocimiento de las diferencias entre el hombre y la mujer. Ello nos sirve para determinar cómo difieren los resultados, experiencias y riesgos sanitarios entre hombres $\mathrm{y}$ mujeres, niños y niñas, y para actuar en consecuencia. Integrar la perspectiva de género en la Salud Pública significa tener en cuenta las diferentes necesidades de la mujer y del hombre en todas las fases del desarrollo de políticas y programas. El objetivo fundamental es lograr la igualdad de género. La incorporación de una perspectiva de género en la Salud Pública implica abordar la influencia de factores picosociales, culturales, y biológicos en los resultados sanitarios, para mejorar así la eficiencia, cobertura y equidad en el acceso a los programas de salud.

Más adelante, la OMS (2009), define la incorporación de la perspectiva de género como el proceso de evaluación de las consecuencias para las mujeres y los hombres de cualquier actividad planificada, incluyendo las leyes, políticas o programas, en todos los sectores y a todos los niveles. Es una estrategia destinada a hacer que las preocupaciones y experiencias de las mujeres, así como de los hombres, sean un elemento integrante de la elaboración, la aplicación, la supervisión y la evaluación de las políticas y los programas en todas las esferas políticas, económicas y sociales, a fin de que las mujeres y los hombres se beneficien por igual y se impida que se perpetúe la desigualdad. El objetivo final es lograr la igualdad entre los géneros.

Introducir la perspectiva de género en el abordaje de los problemas de salud requiere la consideración de las diferencias en la socialización de mujeres y hombres, las cuales determinan distintos valores, actitudes y conductas, las desigualdades en el poder y en el acceso a los recursos y la profunda división sexual del trabajo. Sin embargo, con frecuencia la introducción de la sensibilidad de género en los estudios de los determinantes sociales de la salud se ha basado en muestras constituidas exclusivamente por mujeres, pero el sexo y el género son también determinantes de la salud de los hombres (Courtenay y Doyal, 2001).

En palabras de Gómez (2002), "la equidad de género en salud consistirá en la eliminación de aquellas desigualdades evitables en las oportunidades de disfrutar de la salud y de no enfermar, incapacitarse o morir por causas prevenibles".

Por su parte, Peiró, Vives y Álvarez (2007), sostienen que es necesario señalar que las políticas de salud también deben tener en cuenta las desigualdades de género, lo que se debe visualizar en la construcción social de los problemas de salud, en la toma de decisiones y en la formulación, implantación y evaluación de las políticas de salud. Ante esto, es posible afirmar que las políticas en salud que busquen equidad tendrán como objetivo reducir o eliminar aquellas diferencias que resultan de factores considerados evitables e injustos; creando igualdad de oportunidades para alcanzar el estado de salud deseable.

Al respecto, Cantero y Gálvez, (2007), confirma: "las diferencias en salud que son evitables son las desigualdades en salud". Es decir, que podemos actuar en función de prevenirlas y promover el derecho a la salud en igualdad de condiciones.

Resulta pertinente concluir este artículo, tomando en consideración que la actuación de los profesionales de la Salud Pública ante la problemática de la desigualdad en salud, debe ser la de buscar elevar el nivel de conciencia de las autoridades, profesionales de la Salud y sociedad en general, acerca de las brechas y los retos existentes, así como también, aumentar los conocimientos acerca de la necesidad de la integración de la perspectiva de género para lograr la equidad en salud, promoviendo nuevas líneas de investigación en salud con enfoque de género. En este sentido, se espera que los aportes que surjan sean útiles para las instancias normativas, investigadores, población en general y contribuyan a acelerar los cambios que urge realizar. 


\section{BIBLIOGRAFÍA}

Aldereguía. H. J, Núñez. J. y Fernández. F. (1995). Salud, mujer y desarrollo. Enfoque epidemiológico de riesgo y estado de salud de la población femenina. La Habana: Editorial de Ciencias Médicas.

Braveran. P, Gruskin. S. (2003). Poverty, equity, human rigths and health. Bull WHO. Borrell. C. y Artazcoz. L. (2008). Las Desigualdades de Género en Salud: Retos para el futuro. Rev. Esp. Salud Pública [revista en la Internet]. [citado 2015 Abr 08]; 82(3): 241-249. Disponible en: http://scielo.isciii. es/scielo.php?script $=$ sci_arttext\&pid $=$ S113557272008000300001\&lng=es.

Cantero y Ruiz. M.T. El Enfoque de Género en la Investigación y la Difusión del Conocimiento. [Citado 2015 Abr 09]. Disponible en: http://www. msssi.gob.es/organizacion/sns/planCalidadSNS/ pdf/equidad/12modulo_11.pdf

Cantero, Ruiz. M.T, Gálvez y Papí. N. (2007). Guía de estadísticas de Salud con Enfoque de Género: Análisis de Internet y Recomendaciones. [citado 2015 Abr 09]. Disponible en: https://mspsi.es/en/ organizacion/sns/planCalidadSNS/pdf/equidad/ guiaEstadisticasSal udEnfoqueGenero.pdf

Castañeda. A. (2007). Reflexiones Teóricas Sobre las Diferencias en Salud Atribuibles al Género. Rev Cubana Salud Pública [revista en la Internet]. [citado 2015 Abr 07]; 33(2): Disponible en: $\quad$ http://scielo.sld.cu/scielo.php?script $=$ sci arttext\&pid=S0864-34662007000200011\&lng=es

Courtenay. WH. (2000). Constructions of masculinity and their influence on men's well- being: a theory of gender and health. Soc Sci Med.

Guarnizo, Herreño y Agudelo. (2008). Equidad de Género en el Acceso a los Servicios de Salud en Colombia. Rev. Salud pública [Serie en Internet]. [citado 09 de abril 2015]; 10 (Suppl 1): 44-57. Disponible en: http://www.scielo.org. co/scielo.php?script $=$ sci_arttext\&pid $=$ S012400642008000600005\&lng=en.

Gómez E. (2002). Equidad, género y salud: retos para la acción. Rev Panam Salud Pública.

Krieger. N. y Genders (2003). Sexes, and health: what are the connections--and why does it matter? Int $\mathbf{J}$ Epidemiol.

Organización Mundial de la Salud. OMS. (2007) Género. [Citado 2015 Abr 09]. Disponible en: http:/www.who.int/topics/gender/es/

Organización Mundial de la Salud OMS. (2007). El Enfoque de Salud Pública basado en el Género. [Citado 2015 Abr 09]. Disponible en: http://www. who.int/features/qa/56/es/

Organización Mundial de la Salud. OMS. (2009). Estrategia para Incorporar el Análisis y las Acciones de Género en las Actividades de la OMS. [Citado 2015 Abr 09]. Disponible en: http://whqlibdoc. who.int/publications/2009/9789243597706_spa_ Text.pdf?ua=1

Organización Panamericana de la Salud. OPS. (S.F). Programa Mujer, Salud y Desarrollo. Equidad de Género en Salud. [Citado 2015 Abr 09]. Disponible en:http://www1.paho.org/Spanish/AD/ GE/GEHFactSheetSpanish.pdf?ua=1

Peiró R, Vives C, Alvarez-Dardet C y Más. R. (2007). $\mathrm{El}$ análisis de políticas con enfoque de género y salud.

Weeks, J. (1998). Sexualidad. Paidos UNAM. México. Whitehead. M. (1992). El concepto y los principios de equidad y salud. DiarioInternacional de Servicios de Salud. 